\title{
Energy Release in Air Showers
}

\author{
Markus Risse* and Dieter Heck \\ Forschungszentrum Karlsruhe, Institut für Kernphysik, 76021 Karlsruhe, \\ Germany
}

\begin{abstract}
A simulation study of the energy released in air due to the development of an extensive air shower has been carried out using the CORSIKA code. The contributions to the energy release from different particle species and energies as well as the typical particle densities are investigated. Special care is taken of particles falling below the energy threshold of the simulation which contribute about $10 \%$ to the total energy deposition. The dominant contribution to the total deposition stems from electrons and positrons from sub-MeV up to a few hundred $\mathrm{MeV}$, with typical transverse distances between particles exceeding $1 \mathrm{~mm}$ for $10 \mathrm{EeV}$ showers.
\end{abstract}

\section{Introduction}

During the shower process initiated by a primary cosmic ray in the atmosphere, in general, only a small fraction of the initial energy reaches the ground as high-energy secondary particles. Instead, most of the primary energy is released in the atmosphere by ionization and excitation of the air molecules. A tiny fraction of order $10^{-4}$ is emitted as fluorescence light. As shown by the Fly's Eye experiment [1] and its successor, the High Resolution Fly's Eye [2], the fluorescence component of an air shower can be used to detect cosmic rays at energies exceeding about $10^{18} \mathrm{eV}$. Also the FD detectors of the Pierre Auger Observatory [3] and planned experiments such as EUSO [4], OWL [5], and Telescope Array [6] are based on this detection technique. In contrast to measurements with particle detectors on ground, the observation of the longitudinal shower curve in the fluorescence light provides calorimetric information and therefore conclusions about the primary energy which are largely independent of the primary particle type and of unknown details of hadronic interactions at these extreme energies.

* Correspondence to: M. Risse (markus.risse@ik.fzk.de) 
In the classical approach of reconstructing the primary energy from the measured data $[7,8]$, the amount of light emitted by the shower at a particular atmospheric depth $X$ (determined from the observables by "backtracing" based on detector calibration, atmospheric corrections and previously performed geometrical reconstruction) is converted to a number of charged particles $N_{c h}$ assuming a mean ionization rate. A dependence of the fluorescence yield, i.e. the fraction of the released energy that is emitted as fluorescence light, on temperature and density is taken into account according to [9]. Finally, $N_{c h}(X)$ is integrated over the path-length and multiplied by a mean ionization loss rate and a correction for missing energy is made. Air shower simulations are invoked to obtain average values for the mean ionization rate and for the correction of the missing energy [7].

For such primary energy determinations, it is assumed that the amount of fluorescence photons produced locally per unit length $d N_{f l} / d l$ is proportional to the local energy release $d E_{\text {rel }} / d X$ in air,

$$
\frac{d N_{f l}}{d l}=y(T, \rho) \cdot \rho_{a i r}(h) \cdot \frac{d E_{r e l}}{d X}
$$

with $\rho_{\text {air }}(h)=d X / d l$ being the atmospheric density, where $X$ and $l$ are measured along the shower axis. The fluorescence yield $y(T, \rho)$ of air (in units of emitted photons per released energy) is a key quantity for evaluating the calorimetric shower energy. It has to be determined by laboratory measurements with individual particles as projectiles [9,10]. Due to quenching effects, $y$ is dependent on environmental conditions. Temperature and density have to be varied according to realistic atmospheric conditions with air as target material, maybe even taking humidity effects into account. A considerable experimental effort is started to determine $y(T, \rho)$ more precisely [11]. Different experiments, some of them at accelerator facilities, with various measuring conditions are being planned. Especially, the assumption of a proportionality between fluorescence light production and deposited ionization energy, which has been justified to some extent by previous fluorescence yield measurements $[9,10]$ will be checked thoroughly.

The role of the total deposited ionization energy for the calculation of fluorescence light makes a precise knowledge of the dominant energy deposit processes in air showers important. In Monte Carlo simulations one has to apply a low-energy cutoff for explicit particle tracking. Here we introduce a method for calculating the total ionization energy loss independently of the particular, applied simulation cutoff.

In this work we investigate the characteristics of the energy release in air showers. For this purpose, the CORSIKA simulation code [12] has been adapted to quantify the contributions of different particle species in an air shower to the 
energy release. The electromagnetic component as the dominating one will be examined in more detail.

Our results are also of importance for the planning and interpretation of laboratory fluorescence yield experiments, as we determine the energy ranges that dominate the ionization energy deposit in air showers.

The plan of the paper is as follows. In Chapter 2 it is described how the energy release is calculated in CORSIKA. This includes the definition of a releasable energy for particles which are discarded in the Monte Carlo process since their kinetic energy is below the simulation energy threshold. In Chapter 3, the longitudinal profile of the total energy release and the contributions of different particle species are discussed. The lateral energy deposit distribution together with the densities of the shower particles are analyzed in Chapter 4. The energy spectra of electrons and positrons and their importance for the total energy release are investigated in Chapter 5.

\section{Calculation of the energy release with CORSIKA}

\subsection{The air shower simulation program CORSIKA}

The Monte Carlo program package CORSIKA [12] is designed to simulate the development of extensive air showers induced by various types of primary particles (photons, hadrons, nuclei ... ) in a wide energy range up to the highest energies. For the particle interactions, external state-of-the-art codes are employed. Electromagnetic interactions are simulated using an adapted version [13] of the EGS4 code [14], which includes the Landau-PomeranchukMigdal effect [15].

To describe hadronic interactions at the energies relevant in this paper, accelerator data have to be extrapolated by several orders of magnitude in energy and into a forward kinematic range unobserved in collider experiments. Therefore, CORSIKA offers a choice of various hadronic interaction models which differ in predictions of certain air shower characteristics. For the present investigation, however, the uncertainty related to the modelling of hadronic interactions turns out to be of minor influence, as the main characteristics of the energy release in showers are determined by electromagnetic interactions. For the calculations presented in the following, the model QGSJET $01[16,17]$ has been employed for hadronic interactions with energies $\mathrm{E}_{\text {lab }}>80 \mathrm{GeV}$, while the GHEISHA routines [18] have been used to treat hadronic collisions at lower energies. 
To reduce the computational effort in $\mathrm{CPU}$ time, the technique of particle thinning [19], including weight limitation [20,21], is applied. More specifically, a thinning level of $10^{-6}$ has been chosen, i.e. no thinning occurs for particles with energies exceeding $10^{-6} E_{0}$, with $E_{0}$ being the primary energy. In case of thinning, the bulk of secondary particles produced in an interaction is discarded, and only "representative" particles are sampled. A weight factor is assigned to the selected particles to keep energy conservation [19]. Upper weight limits of $10^{-6} E_{0} / \mathrm{GeV}$ for electromagnetic particles and $10^{-8} E_{0} / \mathrm{GeV}$ for muons and hadrons have been chosen to keep artificial fluctuations introduced by individual particles sufficiently small [22] for the analyses presented in this paper.

In CORSIKA simulations, an (adjustable) energy threshold $E_{t h r}$ is adopted for the shower calculation, i.e. particles are followed explicitely for $E>E_{t h r}$ and discarded for smaller energies. This is due to the fact that a detailed calculation down to smallest energies, for instance to the eV-range, seems both hardly possible (CPU time) and hardly necessary for "classical" air shower experiments measuring surviving particles on ground. In the realm of fluorescence light, however, low-energy particles also contribute to the ionization and excitation of air molecules. Therefore, two categories of shower particles are distinguished for calculating the energy release:

- particles above simulation threshold that are tracked in detail (section 2.2),

- particles below the simulation threshold that are discarded from explicit tracking in the further simulation process (section 2.3).

The final energy release $d E_{r e l} / d X$ is the sum of these two contributions, which will be discussed in the following.

\subsection{Ionization by explicitely tracked charged particles}

The continuous ionization energy loss $d E_{i} / d x$ of a single charged hadron or muon traversing matter of thickness $d x$ along its track is calculated by the Bethe-Bloch stopping power formula

$$
\frac{d E_{i}}{d x}=\frac{z^{2}}{\beta^{2}} \kappa_{1}\left(\ln \left(\gamma^{2}-1\right)-\beta^{2}+\kappa_{2}\right)
$$

where $\beta=v / c$ is the velocity of the particle in the laboratory in units of the velocity of light, $\gamma$ is its Lorentz factor, and $z$ is the charge of the ionizing particle in units of $e$. The two constants $\kappa_{1}=0.153287 \mathrm{MeV} \mathrm{g}^{-1} \mathrm{~cm}^{2}$ and $\kappa_{2}=$ 9.386417 are derived from the tables [23] for dry air. Additionally the EGS4 routines modified for CORSIKA take into account the pressure dependent 
Sternheimer correction [24].

The transport of electrons and positrons with ionization energy loss is treated within CORSIKA in great detail by the EGS4 routines [14]. The contributions of the continuous energy loss stem from the soft bremsstrahlung of photons below the simulation threshold and from sub-threshold energy transfer, including ionization energy loss, to atomic electrons. For the latter contribution, EGS4 uses the formulae recommended by Berger and Seltzer [25] to apply the concept of "restricted stopping power" [26].

\subsection{Treatment of particles below the simulation threshold}

The amount of fluorescence light produced by sub-threshold particles depends on the reactions the specific particle would suffer on its further way, and therefore on the particle type. Antiparticles, for instance, will annihilate and may finally release a much larger amount of energy than the (usually quite low) kinetic energy. Therefore, it is useful to define, similar to the concept of restricted stopping power, the quantity releasable energy $E_{r}$ for each particle species. This releasable energy consists at least of the kinetic energy of the particle. Its contribution to the total energy release is added during the simulation process at the position in air where the particle is discarded.

Stable particles such as electrons cannot release more than their kinetic energy $\left(E_{r}=E_{k i n}\right)$. Due to annihilation, positrons have a larger releasable energy $\left(E_{r}=E_{k i n}+2 \cdot m_{e}=E_{k i n}+1.022 \mathrm{MeV}\right)$. However, if the resulting pair of annihilation photons is above the photon energy threshold, no energy deposition takes place but the photons are treated explicitely in the further simulation process.

Muons and mesons are unstable and can release also part of their rest mass into ionization. Some part will be carried away by the decay neutrinos, however. Antibaryons are assumed to annihilate with a nucleon under emission of several pions, so their releasable energy, apart from $E_{k i n}$, is increased by the antibaryon rest mass and nucleon rest mass. In case of unstable particles and the antibaryons, we assumed an effective fraction of about $1 / 3$ of the releasable energy to be taken into account for the total energy release, and the remaining $2 / 3$ to be "lost" mainly in the neutrino channel. The simplified treatment of these particle species below the simulation energy threshold seems justified as only the contribution of electrons and positrons below threshold turns out to be significant for the present analysis.

The assumption of locality of the energy deposited by the sub-threshold particles is valid for conventional threshold settings in the simulation [22]. While, for instance, a usual choice of the electron energy threshold is about $0.1 \mathrm{MeV}$, 


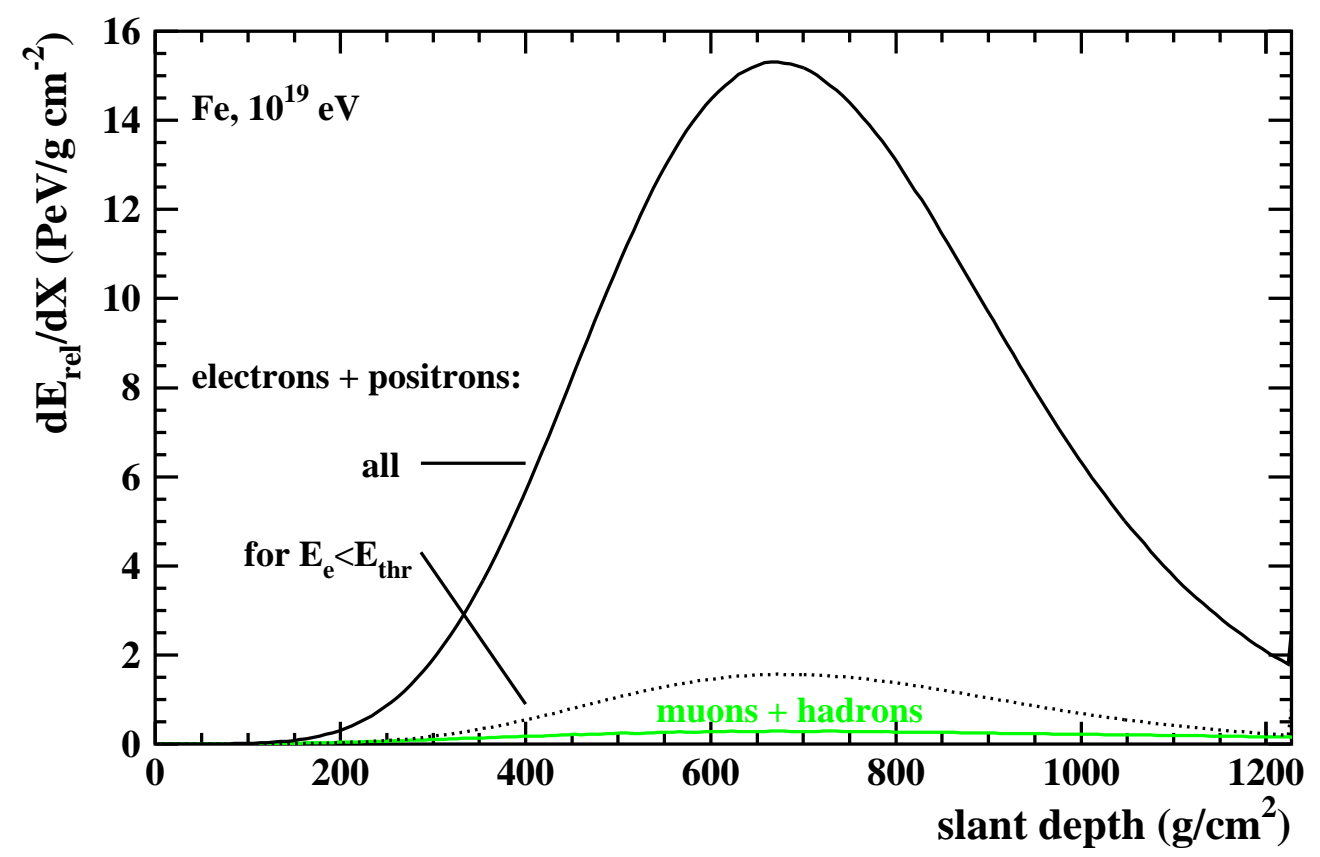

Fig. 1. Longitudinal shower development: Energy release of electrons plus positrons and of muons plus hadrons. Additionally, the contribution of electromagnetic particles below the simulation energy threshold $E_{t h r}=0.1 \mathrm{MeV}$ is given.

even for relatively large threshold values in the $\mathrm{MeV}$ range the particles would be stopped within a depth of only a few $\mathrm{g} \mathrm{cm}^{-2}$.

\section{Longitudinal development}

Air showers initiated by different primary particle types (proton, iron nuclei, and photons) have been calculated with various primary energies $\left(10^{18}-\right.$ $10^{20} \mathrm{eV}$ ) and shower zenith angles. The main conclusions discussed in the following, however, are largely independent of the primary parameter choice and also of the shower-to-shower fluctuations (see also $[27,28]$ ). The distributions given in the Figures illustrate the case of an iron-induced event of primary energy $10^{19} \mathrm{eV}$ with $45^{\circ}$ inclination.

The longitudinal development of the energy release $d E_{r e l} / d X$ of the shower in air is presented in Figure 1. The definition of the path length $d X$ deserves explanation. Plotted is the energy release in the layer of air between slant depths $X$ and $X+d X$, i.e. in the direction of the shower axis. A particle propagating with a non-zero angle $\vartheta$ towards the shower axis thus travels through an effective amount of matter of $d x=d X / \cos (\vartheta)$ while traversing this slant depth interval. The corresponding larger energy loss, or equivalently 
the increased total tracklength of the shower particles due to their angular spread, is taken into account in CORSIKA. This effect has also been pointed out recently in [29].

In Figure 1, different contributions to the energy release are displayed. As expected, the main contribution stems from electrons and positrons, the most numerous charged particles. Around shower maximum, less than 2-3\% are resulting from muons and hadrons. Thus, electromagnetic particles should be the main target for the study of energy release.

A fraction of about $10 \%$ to the energy release is due to discarded electrons and positrons below a simulation energy threshold of $0.1 \mathrm{MeV}$. This value is in agreement with findings in [7]. High-energy electrons produce via Møller scattering a significant number of low-energy electrons. Additionally, the many low-energy shower photons transfer a considerable energy fraction by Compton scattering to low-energy electrons.

A closer inspection shows that the development of the hadronic and muonic parts differ somewhat from the electromagnetic ones: At very early (and also late) development stages the electromagnetic fraction to the local energy release is decreased. For the current analysis, this is of minor interest, however, as the fluorescence light production at these shower stages is marginal compared to the maximum region.

It is important to point out that the shower profile of the total energy release is not influenced when varying the simulation energy threshold within reasonable limits, as also shown in [22]. Adopting, for instance, higher thresholds would increase the contribution of the discarded particles, but this would just be balanced by a decreased contribution of the explicitely tracked particles.

\section{Lateral spread and particle densities}

For laboratory measurements of the fluorescence yield, the question arises whether the particle densities in air showers are small enough to allow for an undisturbed de-excitation or whether an ionized or excited air molecule might be influenced by another nearby shower electron. For this reason, the lateral spread of the energy release and the corresponding density of electrons and positrons as the component which dominates the energy release, is studied. More detailed investigations on the lateral distribution of the energy release in showers including the possibility of observing the shower width with fluorescence telescopes are given elsewhere [30]; here we focus on questions relevant to laboratory measurements of the fluorescence yield. 

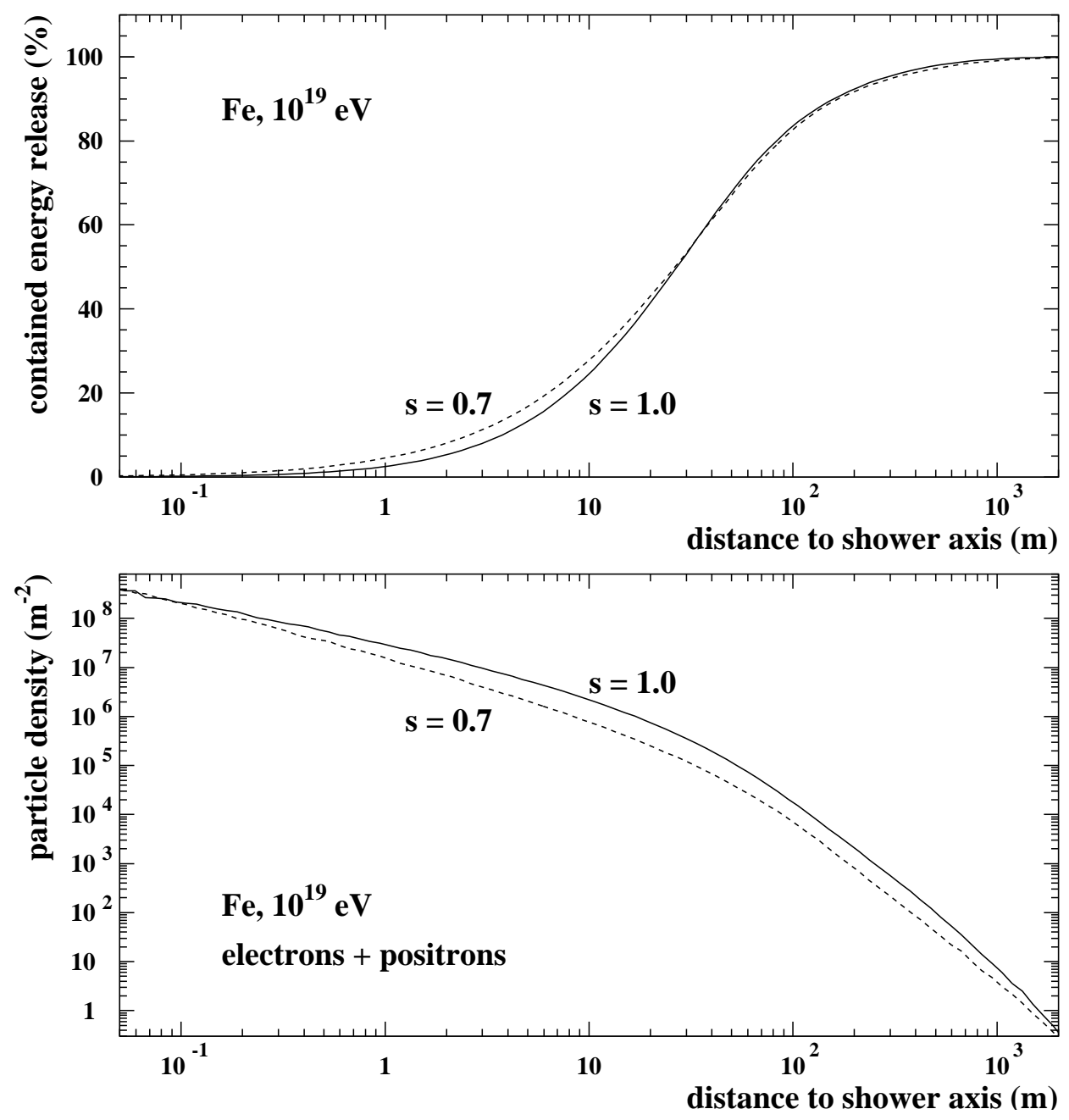

Fig. 2. Upper panel: Energy release contained within a given distance of the shower axis versus the distance at shower ages $s=0.7$ and $s=1.0$. Lower panel: Lateral distribution of electrons plus positrons.

In Figure 2 the amount of released energy contained within a given distance to the shower axis is plotted as a function of the distance (upper panel) for different values of the shower age $s$, defined as $s=3 X /\left(X+2 X_{\max }\right)$. Also given is the density in particles per square-meter of electrons and positrons (lower panel). As the lifetime of the radiating molecular states is of the order 30-70 ns and thus comparable to the traversal time of the shower for a given air particle (or correspondingly to the shower "thickness"), transverse particle distances are regarded for a conservative estimate of the particle separation.

Particles with core distances below $1 \mathrm{~m}$ contribute only little to the energy release: Though the particle densities are largest here, due to phase space the absolute particle number is comparatively small. The main energy release of about $80-85 \%$ occurs at distances between $1-100 \mathrm{~m}$ from the shower 
axis. For core distances around $20 \mathrm{~m}$, a typical transverse particle separation of $\simeq 1 \mathrm{~mm}$ for $10 \mathrm{EeV}$ showers is obtained. This value holds for the shower maximum, at other stages of the shower development observable in the flourescence light the particle separations are larger. The densities are roughly scaling with the primary energy, while the dependence on the primary particle type is of minor importance in this context. For $100 \mathrm{EeV}$ showers at relatively small core distances of about $3 \mathrm{~m}$, for instance, the average transverse particle separation amounts to $\simeq 0.1 \mathrm{~mm}$. Thus, with respect to the size of the ionization region around the charged particles in air (usually $<1 \mu \mathrm{m}$ ), this is a large separation resulting in a relatively "undisturbed" de-excitation of the air molecules. High-density particle bunches should therefore be avoided in fluorescence yield measurements as the fluorescence yield, apart from extreme cases of high shower particle densities, would be obtained in conditions not typical for fluorescence light emission in air showers.

\section{$5 \quad$ Energy spectra}

The particle energies contributing to the energy release, an important input quantity for the layout of fluorescence yield measurements, are shown in Figure 3. For this graph, only electrons and positrons are taken into account, since they dominate the energy release. The main contribution comes from particles with energies below $1 \mathrm{GeV}$, with a tail towards small energies. A broad maximum is visible at particle energies around $10-50 \mathrm{MeV}$, below the critical energy of electrons in air $(\simeq 84 \mathrm{MeV})$.

The spectral shape mainly reflects the particle energy spectrum [21]. Especially the contributions of the lower energies are more pronounced, however. This is due firstly to the increased specific energy loss (Bethe-Bloch formula), and secondly to a larger average path length $d x=d X / \cos (\vartheta)$ through the slant depth interval $d X$, since at lower electron energies the dispersion of particle angles is increasing. As a guideline, in Table 1 the contribution to the electromagnetic energy release around shower maximum is estimated for different ranges in kinetic energy. The value for $E_{k i n}<0.1 \mathrm{MeV}$ is given by the contribution of the particles discarded due to the simulation energy threshold.

Table 1

Estimates for the contribution of different ranges in kinetic energy to the energy release by electrons and positrons. The uncertainty of the values is about \pm 2 (in \%).

\begin{tabular}{|c||c|c|c|c|c|c|}
\hline Energy in $\mathrm{MeV}$ & $<0.1$ & $0.1-1$ & $1-10$ & $10-100$ & $100-1000$ & $>1000$ \\
\hline Contribution in \% & 10 & 12 & 23 & 35 & 17 & 3 \\
\hline
\end{tabular}



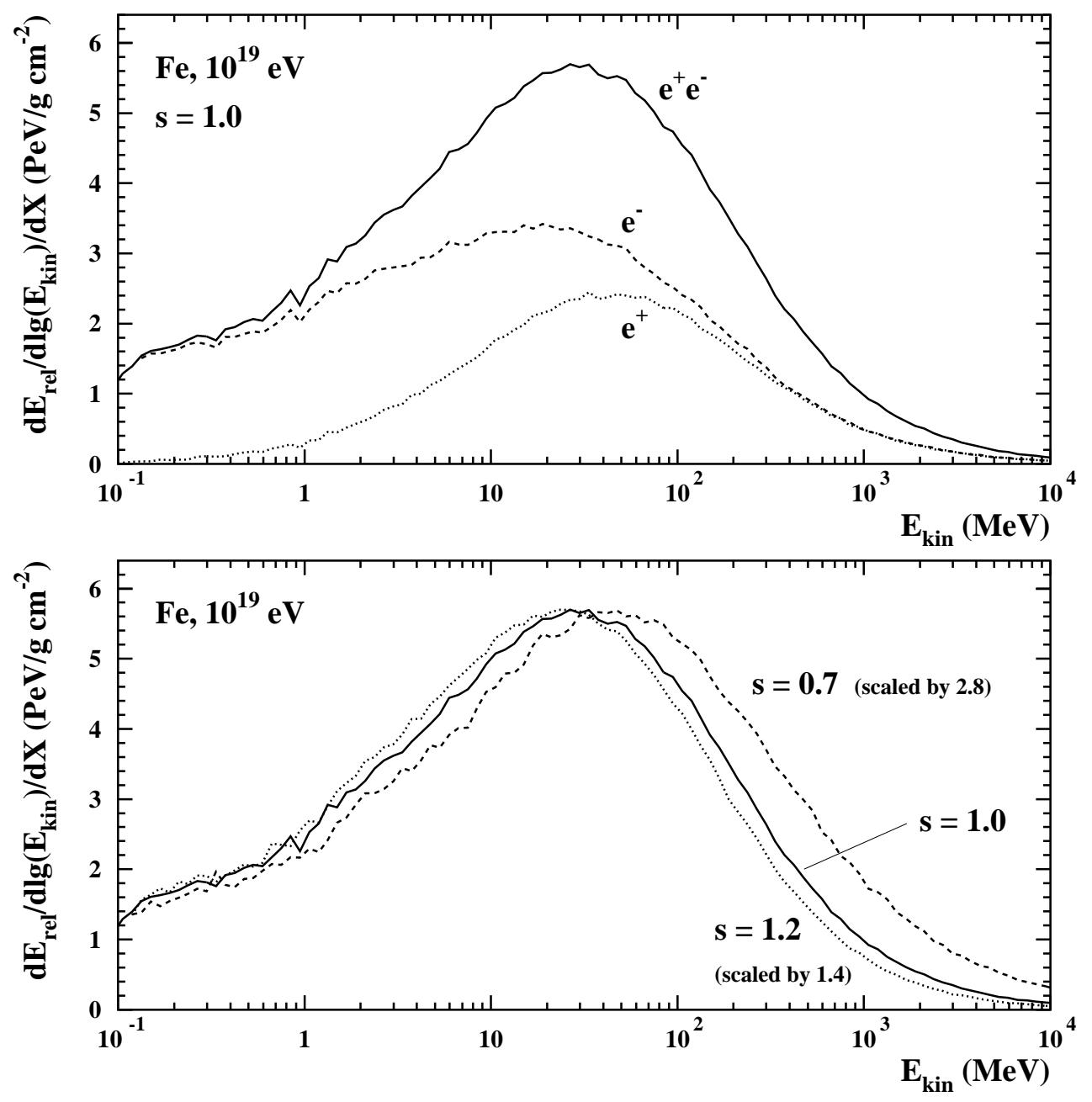

Fig. 3. Contribution to the energy release per matter traversed in shower direction as a function of the kinetic particle energy. Simulation for primary iron, $10^{19} \mathrm{eV}$. Upper panel: Individual and combined contributions of electrons and positrons at shower maximum. Lower panel: Combined contribution of electrons and positrons for different shower ages (normalized to the same height of maximum).

While at higher kinetic energies $\left(E_{k i n}>300 \mathrm{MeV}\right)$ electrons and positrons contribute about equally to the energy release, at lower energies only electrons survive due to positron annihilation. The annihilation photons will eventually transfer the energy to electrons by Compton scattering.

The range of particle energies that mainly contribute to the energy release, is to a good approximation quite independent of the primary particle type (including primary photons) and primary energy. Also the dependence on shower age is small, as shown in the lower panel of Figure 3. For instance, at earlier development stages before the shower maximum, the contribution is only slightly shifted to higher electron energies. These results may be understood, since the 
particle energy spectrum is known to show a small, but in this context only minor dependence on the primary particle type and on shower age $[21,28]$.

\section{Conclusion}

The presented method of treating sub-threshold particles allows the precise calculation of the total energy deposit. For shower calculations, the energy release provided by CORSIKA can be transformed to fluorescence light based on existing and upcoming fluorescence yield measurements.

The energy release in air showers has also been studied with respect to currently planned laboratory fluorescence yield measurements. Most relevant is the determination of the yield for electrons and positrons with energies in the range from sub-MeV up to a few hundred $\mathrm{MeV}$. The typical lateral particle separation is relatively large with $1 \mathrm{~mm}$ or more for $10 \mathrm{EeV}$ showers at shower distances where most energy is released and thus, presumably, the main fluorescence light production occurs.

Finally, we want to mention that based on the concept of total shower size one can define the mean energy deposit per particle [1,7]. This quantity has been subject of many discussions and will be studied in detail in a forthcoming publication.

Acknowledgements. The useful discussions with D. Góra, H. Klages, J. Knapp, P. Sommers, and T. Waldenmaier and in particular with R. Engel are gratefully acknowledged.

\section{References}

[1] R.M. Baltrusaitis et al., Nucl. Instr. Meth. A240 (1985) 410

[2] T. Abu-Zayyad et al., Nucl. Instr. Meth. A450 (2000) 253

[3] J. Abraham et al., Auger Collaboration, submitted to Nucl. Inst. Meth. (2003)

[4] L. Scarsi et al., $27^{\text {th }}$ Int. Cosmic Ray Conf., Hamburg 2 (2001) 839

[5] L. Scarsi et al., 26 ${ }^{\text {th }}$ Int. Cosmic Ray Conf., Salt Lake City 2 (1999) 384

[6] T. Aoki et al., $27^{\text {th }}$ Int. Cosmic Ray Conf., Hamburg 2 (2001) 915

[7] C. Song, Z. Cao, B.R. Dawson, B.E. Fick, P. Sokolsky, and X. Zhang, Astropart. Phys. 14 (2000) 7 
[8] T. Abu-Zayyad et al., HiRes Collaboration, submitted to Astropart. Phys. (2003); preprint astro-ph/0208301 (2002)

[9] F. Kakimoto, E.C. Loh, M. Nagano, H. Okuno, M. Teshima, and S.Ueno, Nucl. Instr. Meth. A 372 (1996) 527

[10] M. Nagano, K. Kobayakawa, N. Sakaki, and K. Ando, Astropart. Phys. (2003) in press; preprint astro-ph/0303193

[11] International workshops in Utah (USA) 2002 and Bad Liebenzell (Germany) 2003; see www.physics.utah.edu/ ${ }^{\sim}$ fiwaf/done and www.auger.de/events

[12] D. Heck, J. Knapp, J.N. Capdevielle, G. Schatz, and T. Thouw, Report FZKA 6019, Forschungszentrum Karlsruhe (1998); www-ik.fzk.de/ ${ }^{\sim}$ heck/corsika

[13] D. Heck and J. Knapp, Report FZKA 6097, Forschungszentrum Karlsruhe (1998)

[14] W.R. Nelson, H. Hirayama, and D.W.O. Rogers, Report SLAC 265, Stanford Linear Accelerator Center (1985)

[15] L.D. Landau and I.Ya. Pomeranchuk, Dokl. Akad. Nauk SSSR 92 (1953) 535 \& 735 (in Russian); A.B. Migdal, Phys. Rev. 103 (1956) 1811

[16] N.N. Kalmykov, S.S. Ostapchenko, and A.I. Pavlov, Nucl. Phys. B (Proc. Suppl.) 52B (1997) 17

[17] D. Heck et al., Proc. $27^{\text {th }}$ Int. Cosmic Ray Conf., Hamburg (Germany) 1 (2001) 233

[18] H. Fesefeldt, Report PITHA-85/02, RWTH Aachen (1985)

[19] A.M. Hillas, Nucl. Phys. B (Proc. Suppl.) 52B (1997) 29

[20] M. Kobal, Pierre Auger Collaboration, Astropart. Phys. 15 (2001) 259

[21] M. Risse, D. Heck, J. Knapp, and S.S. Ostapchenko, Proc. $27^{\text {th }}$ Int. Cosmic Ray Conf., Hamburg (Germany), 2 (2001) 522

[22] M. Risse and D. Heck, Auger Internal Note GAP-2002-043, www.auger.org, (2002)

[23] R.M. Sternheimer, M.J. Berger and S.M. Seltzer, Atomic Nucl. Data Tables 30 (1984) 261

[24] R.M. Sternheimer et al., Phys. Rev. B26 (1982) 6067

[25] M.J. Berger and S.M. Seltzer, Report NASA-SP-3012 (1964)

[26] K.R. Kase and W.R. Nelson, Concepts of Radiation Dosimetry, Pergamon Press, New York (1979)

[27] M. Risse and D. Heck, Proc. 28 ${ }^{\text {th }}$ Int. Cosmic Ray Conf., Tsukuba (Japan) (2003) 607 
[28] F. Nerling, R. Engel, C. Guerard, L. Perrone, and M. Risse, Proc. $28^{\text {th }}$ Int. Cosmic Ray Conf., Tsukuba (Japan) (2003) 611

[29] J. Alvarez-Muñiz, E. Marqués, R.A. Vázquez, and E. Zas, Phys. Rev. D 67 (2003) 101303

[30] D. Góra, P. Homola, M. Kutschera, J. Niemiec, B. Wilczyńska, and H. Wilczyński, Astropart. Phys. 16 (2001) 129; D. Góra et al., in preparation (2003) 